initiatives. Methodology: A grant was obtained from the Coalition for a Healthier Community based on a 2006 HCC assessment data. A recent community needs assessment was conducted in 2010 using cross-sectional techniques. Data were collected and analysed from a random sample of community members. The HCC assessment data were analysed to evaluate mental health among women 18 years of age and older.

Results Eleven per cent of women stated that they had experienced intense stress, depression, and/or difficulty managing your emotions for at least 2 weeks or more. Approximately, $10 \%$ of women stated that they sought assistance from a counsellor for mental health purposes. Another $6 \%$ stated that they had difficulty obtained needed mental healthcare within the community. Women (10\%) in the community identified family counselling as their main need among a list of services. Social support networks were also assessed. Women stated that they turned to family (73\%), friends (59\%) and a spouse or partner $(48 \%)$ in times of need.

Conclusions Data from the HCC assessment on mental health of women in the community can be utilised to identify needs and establish priorities. The data are beneficial in planning for mental health services within the community.

\section{P2-153 PRENATAL STRESS FOLLOWING MATERNAL BEREAVEMENT DURING PREGNANCY AND OVERWEIGHT IN THE OFFSPRING: A COHORT STUDY IN 109919 DANISH CONSCRIPTS}

doi:10.1136/jech.2011.142976i.88

\section{J Li, ${ }^{*}$ C Obel, M Vestergaard, J Olsen. Aarhus University, Aarhus, Denmark}

Introduction It has been suggested that prenatal stress contributes to the risk of obesity later in life. In a population-based cohort study, we examined whether prenatal stress related to maternal bereavement during pregnancy was associated with the risk of overweight in the offspring in young adulthood.

Methods We conduct a cohort study of 109919 Danish men who were born in 1986-1990 and presented for conscription in 2006-2009. By linkage of the conscription file ("session") and national registers, we identified 4549 conscripts who were exposed to prenatal stress, defined by being born to mothers who were bereaved by death of a close family member from 1 year before pregnancy until birth of the child. Differences in the means in Body mass index and RR of overweight between the exposed and the unexposed were analysed by GLM regression or Logistic regression in SAS.

Results Body mass index values and prevalence of overweight were higher in the exposed group. The overall adjusted OR for overweight was 1.13 (95\% CI 1.04 to 1.23). The highest OR was observed in the subjects whose mothers lost a child or husband during pregnancy (OR 1.35, 95\% CI 1.07 to 1.71 ).

Conclusions Our results suggest that severe pre-pregnancy stress is associated with an increased risk of overweight in the offspring in young adulthood.

\section{P2-154 "TRIGLYCERIDEMIC-WAIST" PHENOTYPE PREDICTS CORONARY HEART DISEASE IN ABORIGINAL AUSTRALIANS}

doi:10.1136/jech.2011.142976i.89

${ }^{1} \mathrm{M}$ Li, ${ }^{2}{ }^{2} \mathrm{~B}$ McCulloch, ${ }^{1} \mathrm{R}$ McDermott. ${ }^{1}$ Division of Health Sciences, University of South Australia, Adelaide, South Australia, Australia; ${ }^{2}$ Tropical Public health Unit, Oueensland Health Cairns, Cairns, Queensland, Australia

Introduction Australian Aboriginal and Torres Strait Islands (TSI) people experience socioeconomic disadvantage and have higher prevalence of risk factors for cardiovascular diseases including overweight and obesity, smoking and alcohol drinking, higher blood triglycerides. In this population, diabetes and albuminuria have been reported to better predict some of the excess incident CHD than the "traditional" Framingham risk factors. This study aims to compare the relative performance of components of the metabolic syndrome (MetS) in predicting coronary heart disease morbidity and mortality in a cohort of Australian Aboriginal and Torres Strait Islander adults.

Methods Cohort study of 2100 adults (1283 Aborigines and 817 TSI) with 7-year follow-up from 2000. Outcome measures were all CHD events (deaths and hospitalisations). Baseline anthropometric measurements, blood pressure, fasting blood lipids and glucose were collected. Alcohol and tobacco intake was by self report.

Results MetS (both ATP-III and IDF criteria) was more prevalent in TSI males (50.5\%) compared to Aborigines (37.7\%). MetS at baseline doubled the risk of a $\mathrm{CHD}$ event in Aborigines. Increased fasting triglycerides was stronger in predicting CHD compared with MetS (HR: 2.8) after adjusted for age, sex, tobacco and alcohol consumption, and baseline diabetes and albuminuria for Aborigines but not among TSIs.

Conclusion Indigenous Australians have a high prevalence of $\mathrm{CHD}$ risk factors. MetS was not more powerful than its components in predicting CHD events. In Australian Aborigines, the "triglyceridemic waist" phenotype best predicts CHD events, while among TSIs, increased fasting glucose was a stronger predictor of $\mathrm{CHD}$.

\section{P2-155 CONSUMPTION OF ULTRA-PROCESSED FOOD IS ASSOCIATED WITH BLOOD PRESSURE IN HYPERTENSIVE INDIVIDUALS}

doi:10.1136/jech.2011.142976i.90

${ }^{1} \mathrm{R}$ Lima, ${ }^{1,2} \mathrm{~L}$ Moreira, ${ }^{1,3} \mathrm{~S}$ Rossato, ${ }^{*} \mathrm{R}$ Silva, ${ }^{1,2} \mathrm{~S}$ Fuchs. ${ }^{1}$ Postgraduate Studies Program in Health Science: Cardiology and Cardiovascular Diseases; UFRGS, Porto Alegre, Rio Grande do Sul , Brazil; '2Division of Cardiology; Hospital de Clínicas de Porto Alegre, Porto Alegre, Rio Grande do Sul, Brazil; ${ }^{3}$ Postgraduate Studies Program in Epidemiology; UFRGS, Porto Alegre, Rio Grande do Sul, Brazil

Introduction The consumption of the ultra-processed food has been associated with a growing prevalence of chronic diseases.

Objective To assess the effect of processed food consumption on systolic (SBP) and diastolic (DBP) blood pressure among hypertensive patients.

Methods This cross-sectional study enrolled 125 patients, aged 30-80 years, followed-up on the outpatient Hypertension Clinic from a reference Center (Hospital de Clínicas de Porto Alegre), in southern Brazil. Food intake was assessed by 24-h dietary recall administered four times one week apart. Food items were aggregated by the types of processing into unprocessed (group 1), culinary foods (group 2), and ultra-processed foods (group 3). The intake was calculated by the number of items per group consumed daily and categorised in quartiles. Generalised Estimated Equation (GEE) was adopted in the analysis.

Results The consumption amount of unprocessed and culinary food was not associated with blood pressure. Among ultraprocessed foods, the comparison between patients in the first and the forth quartile showed a difference of $15.8 \pm 4.0 \mathrm{~mm} \mathrm{Hg}$ in $\operatorname{SBP}(p<0.01)$ and $6.3 \pm 2.3$ in DBP $(p=0.02)$, controlled for age, gender and the consumption of both unprocessed and culinary food. No difference was observed for the $2^{\text {nd }}$ and $3^{\text {rd }}$ quartiles vs de $1^{\text {st }}$ one.

Conclusion The ultra-processed foods consumption can affect SBP and DBP of hypertensive individuals. 